\title{
3 Research Square

\section{Clinical and Histological Features of Prosthetic Joint Infections May Differ In Patients With Inflammatory Arthritis and Osteoarthritis}

\section{Peter Sculco}

Hospital for Special Surgery

Milan Kapadia

Hospital for Special Surgery

Carine Moezinia

Hospital for Special Surgery

Andy Miller

Hospital for Special Surgery

Laura Donlin

Hospital for Special Surgery

Michael Henry

Hospital for Special Surgery

Linda Russell

Hospital for Special Surgery

Mark Figgie

Hospital for Special Surgery

Allina Nocon

Hospital for Special Surgery

Tania Pannellini

Hospital for Special Surgery

Susan Goodman ( $\sim$ goodmans@hss.edu )

Hospital for Special Surgery

\section{Research Article}

Keywords: inflammatory arthritis, periprosthetic joint infection, total hip arthroplasty, total knee arthroplasty, culture negative, histopathology

Posted Date: January 27th, 2021

DOl: https://doi.org/10.21203/rs.3.rs-147768/v1 
License: (c) (i) This work is licensed under a Creative Commons Attribution 4.0 International License. Read Full License 


\section{Abstract}

Background: Inflammatory arthritis (IA) patients are at increased risk for prosthetic joint infections (PJI); however, diagnosis is challenging because active IA patients have elevated inflammatory markers that may mimic joint infection and definitive microbiologic diagnosis may be delayed. The objective of this pilot study was to identify the clinical, microbiologic, and histopathologic features of culture positive (CP) and culture negative (CN) total hip (THA) and total knee arthroplasty (TKA) PJI in IA patients and obtain preliminary data to support a definitive study to determine the optimal method for PJI diagnosis in patients with inflammatory arthritis.

Methods: A retrospective cohort of THA/TKA PJIs, from 2009 to 2016, were identified using an institutional PJI registry. H\&E slides of OA and IA PJI cases matched by age, sex, and culture status were histologically reviewed. Clinical characteristics were evaluated using Fisher's exact, Chi-Square tests, Students' t test, and Mann Whitney U test where appropriate.

Results: 807 PJI cases were identified including 36 IA and 771 OA. A higher proportion of IA PJI were CN $(\mathrm{N}=10,27 \%)$ vs. OA PJI $(\mathrm{N}=109,14 \%, \mathrm{p}=0.02)$. IA PJI patients compared to OA patients presented younger $(p<0.001)$, with a higher Charlson Comorbidity Index $(p=0.002)$, had more frequent use of glucocorticoids $(p<0.001)$ and a larger proportion were female $(p<0.001)$. Of 88 PJIs reviewed for histopathology, within the IA cohort, a higher proportion of CP-IA had $>10$ PMN per HPF $(p=0.003)$ and met MSIS criteria $(p=0.009)$ but presented with less chronic inflammation $(p=0.017)$.

Conclusions: This hypothesis-generating study suggests that culture negative PJI is more frequent in patients with IA than OA. Higher rates of prior PJI, differences in histopathology, and better clinical outcomes suggest biologic differences between CN and CP PJI that should be explored further in a prospective study.

\section{Background}

Approximately one million total hip (THA) and total knee arthroplasties (TKA) are performed in the USA each year and effectively decrease pain and improve function for patients with advanced symptomatic arthritis. ${ }^{1,2}$ A relatively rare but devastating complication is prosthetic joint infection (PJI) which complicates $0.8-1.9 \%$ of TKAs and is the most common cause of TKA failure and is the third most common cause of THA failure, affecting $0.3-1.7 \%$ of patients. ${ }^{3}$ The risk of PJI after TKA or THA has been reported to be $50-80 \%$ higher for patients with inflammatory arthritis (IA) conditions such as rheumatoid arthritis (RA) and systemic lupus erythematosus (SLE). ${ }^{4-6}$ Epidemiologic studies have identified differences in the onset of PJI between osteoarthritis (OA) and RA patients, namely OA patients are at highest risk for PJI within the first two years of surgery whereas the risk of PJI persists for the life of the implant in RA patients. ${ }^{7}$ The increased risk of PJI in IA patients may be related pharmacologic immunosuppression, immune dysregulation, and/or high disease activity. ${ }^{8-10}$ However, the diagnosis of $\mathrm{PJI}$ in patients with IA is challenging because aseptic RA or SLE flares may mimic PJI both clinically (joint 
pain, swelling, fever) and diagnostically (elevated C-reactive protein (CRP), erythrocyte sedimentation rate (ESR), and synovial leukocytosis). ${ }^{11}$ In addition, the currently available diagnostic criteria for diagnosing PJI does not provide guidance on how to differentiate PJI from RA flares and few studies have comprehensively characterized PJI in patients with RA and SLE.

We hypothesized that the clinical and diagnostic overlap between flares of IA and PJI suggests that flares may contribute to the higher rate of "culture negative" PJI observed in IA patients. For this reason, more information is needed to better diagnose and treat PJI patients with IA. The purpose of this study is to describe the clinical, microbiological, and histopathologic features of surgically managed THA and TKA culture positive and culture negative PJI in patients with IA and OA using an institutional PJI database to explore our hypothesis. Our aim was to obtain pilot data from this retrospective cohort to use in the design of a future prospective study.

\section{Methods}

A retrospective analysis from 2009 to 2018 of TKA and THA patients treated for PJI at a single tertiary care orthopedic institution using a longitudinally maintained hospital infection database was performed. Patients were included if they had an ICD code denoting a diagnosis of PJI and the presumed PJI was managed surgically in conjunction with an Infectious Disease specialist. Inclusion criteria required at least two years of clinical and radiographic follow-up. Surgical treatment consisted of 1. Debridement, Antibiotics, and Implant Retention (DAIR), 2. two-stage exchange or 3. one-stage exchange followed by at least six weeks of IV antibiotics in all cases. IA patients were defined as any patient with an ICD diagnosis code for rheumatoid arthritis (ICD-10 M05-), systemic lupus erythematosus (ICD 10-M32-), or another IA who received a prescription for a disease modifying anti-rheumatic drug (DMARD), biologic, or SLE-

specific medications within 1 year of surgery. ${ }^{8,12}$ Patients with no diagnosis of IA were considered to have OA. Culture negative $(\mathrm{CN})$ patients were defined as any patient with negative culture growth either intra-op and/or negative pre-operative aspirate culture growth. ${ }^{9,10}$ For those who had culture mismatch (aspiration and intraoperative finding did not agree), infection disease specialist confirmed infection status. Culture positive (CP) patients had at least one intraoperative or preoperative aspirate culture growing any microorganism. Additional collected information included MSIS major and minor criteria, prior history of orthopedic infection, chronic glucocorticoid use, type of DMARD, tobacco use and Charlson comorbidities which were all abstracted from the hospital record.

Hematoxylin and eosin (H\&E) stained slides of OA and IA PJI cases, matched by age (+/-5), sex, and culture status, were reviewed by an experienced musculoskeletal pathologist for histological evidence of infection. Following AAOS guidelines, a polymorphonuclear leucocyte (PMNs) count $\geq 10$ per 5 high power fields was considered positive histological evidence of infection. The 5 fields were selected as the busiest in the section, and, when possible, non-consecutive. Chronic inflammation was reviewed, according to the following scoring parameters: absent chronic inflammation (0), scattered chronic inflammatory cells (1), at least one lymphoid aggregate in 2 separate fields (2), and more than one aggregate in two separate fields, or band like pattern (3). Tissue necrosis containing PMNs was reviewed 
according to the following parameters: absent (0), small rare micro-abscesses (1), visible areas of necrosis with purulent material (2), and excessive purulent necrosis (3) (Fig. 1). All patients were treated with six weeks of intravenous antibiotics prescribed by an infectious disease specialist. In addition, patients who retained hardware (DAIR) after irrigation and debridement were prescribed at least six months of suppressive oral antibiotic therapy. Treatment success was defined as no further surgical intervention for infection recurrence within two years of the original PJI treatment. ${ }^{1,2}$

DAIR procedures involved complete synovectomy and exchange of modular components. Two-stage revision procedures consisted of initial removal of components, total knee or hip replacement bone resection cuts, placement of a high-dose antibiotic spacer, 6 weeks of intravenous or oral antibiotics followed by minimum 2-week antibiotic holiday, and definitive prosthetic joint insertion once reassessment for persistent infection was complete. One-stage revision procedures consisted of complete synovectomy, removal of components, total knee or hip replacement cuts, preliminary wound closure with re-sterilization and draping, and placement of definitive prosthesis components in the same operative setting. ${ }^{3}$

Institutional review board approval was waived for this retrospective study prior to study initiation.

\section{Statistical Analysis}

Categorical data was analyzed with Fischer exact square tests or the chi-square test and continuous data was analyzed using a student $t$ test or Mann-Whitney $U$ tests based on data distribution. Kaplan-Meier survival curves were constructed to analyze the survivorship free of reoperation due to infection. ${ }^{13} 95 \%$ confidence intervals $(95 \% \mathrm{Cls})$ were calculated for survival curves. Significance was set at alpha $<0.05$. Statistical analyses were performed using SAS 9.4 (SAS Institute Inc., Cary, NC).

\section{Results}

A total of 807 PJI cases were identified, including 36 IA cases (33 had RA and 3 had SLE) and 771 OA cases. For the overall PJI cohort (807 cases), the rate of culture negative PJI was $14.7 \%$. The rate of culture negative cases was significantly higher $(p=0.024)$ in IA patients $(27 \%, n=10)$ compared to OA patients $(14 \%, n=109)$. Compared to OA patients, IA PJI patients were younger $(p<0.001)$, had a higher Charlson Comorbidity Index $(p=0.002)$, and more frequent use of glucocorticoids $(p<0.001)$ (Table 1$)$. 
Table 1

Patient characteristics in inflammatory arthritis and osteoarthritis PJIs

\begin{tabular}{|c|c|c|c|c|c|}
\hline & IA & & OA & & \\
\hline & $\mathbf{N}$ & $\% / S D$ & $\mathbf{N}$ & $\% / S D$ & $\mathrm{p}$-value \\
\hline Total & 36 & & 771 & & \\
\hline Age & 58.5 & 11.4 & 66.8 & 12 & $<.001$ \\
\hline BMI & 30.2 & 6.7 & 30 & 6.7 & 0.861 \\
\hline Female & 28 & 77.8 & 332 & 43.1 & $<.001$ \\
\hline Joint & & & & & 0.999 \\
\hline Hip & 16 & 44.4 & 347 & 45 & \\
\hline Knee & 20 & 55.6 & 424 & 55 & \\
\hline $\mathrm{CCl}$ & 2.8 & 1.7 & 1.7 & 2.1 & 0.002 \\
\hline History of Smoking & 4 & 11.1 & 86 & 11.2 & 0.792 \\
\hline Glucocorticoids & 10 & 27.8 & 39 & 5.1 & $<.001$ \\
\hline Culture Negative & 10 & 27.8 & 109 & 14.1 & 0.024 \\
\hline Surgical Therapy & & & & & 0.91 \\
\hline One Stage Exchange & 2.8 & 33 & 4.3 & & \\
\hline Two Stage Exchange & 18 & 50 & 378 & 49 & \\
\hline DAIR & 17 & 47.2 & 360 & 46.7 & \\
\hline Treatment Success at 2 years & 19 & 52.8 & 509 & 66 & 0.146 \\
\hline
\end{tabular}

The type of surgical treatment used and treatment success at 2 years did not differ between the IA and OA group. The study cohort was then subdivided into four groups for further statistical comparison of IA and culture positive (IA-CP), $N=26$; IA and culture negative (IA-CN), $N=10 ; O A$ and culture positive (OA$\mathrm{CP}), \mathrm{N}=662$; and $\mathrm{OA}$ and culture negative (OA-CN), $\mathrm{N}=109$. Histopathology of 88 PJIs (31 IA and $57 \mathrm{OA}$ ) was reviewed. Histological signs of high chronic inflammation (scores 2 or 3 ) were significantly higher in the IA group $(74 \%)$ compared to OA cohort $(23 \%, \mathrm{p}<0.001)$ (Tables 2 and 3$)$. 
Table 2

Patient Characteristics in inflammatory arthritis PJIs

\begin{tabular}{|c|c|c|c|}
\hline & $\mathrm{CN}-\mathrm{IA}(\mathrm{N}=10)$ & CP-IA $(N=26)$ & \\
\hline & N(\%)/Mean(SD & & p-value \\
\hline Age & $59.04(10.21)$ & $58.35(12.02)$ & 0.986 \\
\hline Body mass index & $25.88(9.81)$ & $27.16(12.23)$ & 0.697 \\
\hline Sex & & & 0.842 \\
\hline Female & $8(80)$ & $20(76.92)$ & \\
\hline Male & $2(20)$ & $6(23.08)$ & \\
\hline Joint & & & 0.285 \\
\hline Knee & $6(60)$ & $10(38.46)$ & \\
\hline Hip & $4(40)$ & $16(61.54)$ & \\
\hline History of Smoking & $1(10)$ & $3(11.54)$ & 1.000 \\
\hline Diabetes & $0(0)$ & $5(19.23)$ & 0.293 \\
\hline History of prior PJI & $2(20)$ & $0(0)$ & 0.071 \\
\hline Glucocorticoids & $2(20)$ & $8(30.77)$ & 0.518 \\
\hline DMARD & & & 0.397 \\
\hline Biologic & $5(50)$ & $9(34.62)$ & \\
\hline Synthetic only & $5(50)$ & $17(65.38)$ & \\
\hline Surgical Therapy & & & 0.664 \\
\hline One Stage Exchange & $0(0)$ & $1(3.85)$ & \\
\hline Two Stage Exchange & $6(60)$ & $12(46.15)$ & \\
\hline DAIR & $4(40)$ & $13(50)$ & \\
\hline Treatment Success at 2 years & $7(70)$ & $12(63.16)$ & 0.199 \\
\hline
\end{tabular}


Table 3

Histopathology and clinical presentation in inflammatory arthritis and osteoarthritis PJls Histopathology and clinical presentation in inflammatory arthritis and osteoarthritis PJls

$\begin{array}{llllll}\mathrm{OA} & \mathrm{IA}(\mathrm{N} & \mathrm{CP}-\mathrm{OA} & \mathrm{CN}-\mathrm{OA} & \mathrm{CP}-\mathrm{IA} & \mathrm{CN}-\mathrm{IA} \\ (\mathrm{N}= & =31) & (\mathrm{N}= & (\mathrm{N}=17) & (\mathrm{N}= & (\mathrm{N}= \\ 57) & & 40) & & 23) & 8)\end{array}$

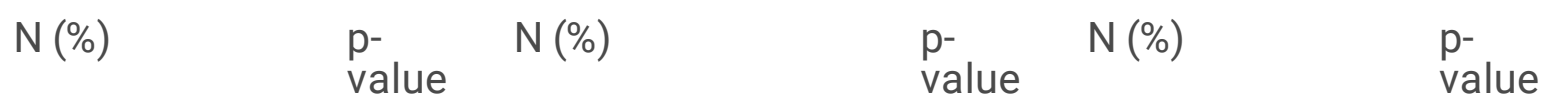

\section{Pathology \\ Review}

$\begin{array}{llllllllll}\begin{array}{ll}210 \text { PMN per } \\ \text { 5HPFs }\end{array} & \begin{array}{l}42 \\ (74)\end{array} & \begin{array}{l}22 \\ (71)\end{array} & 0.806 & 30(75) & 12(71) & 0.785 & \begin{array}{l}20 \\ (87)\end{array} & 2(25) & 0.003 \\ \begin{array}{lllllll}\text { Chronic } \\ \text { Inflammation }\end{array} & \begin{array}{l}13 \\ (23)\end{array} & \begin{array}{l}23 \\ (74)\end{array} & \mathbf{0 . 0 0 1} & 11(28) & 2(12) & 0.412 & \begin{array}{l}18 \\ (78)\end{array} & 5(63) & 0.393 \\ \text { Necrosis } & \begin{array}{l}17 \\ (30)\end{array} & \begin{array}{l}9 \\ (29)\end{array} & 0.999 & 13(33) & 4(24) & 0.938 & 8(35) & 1(13) & 0.38 \\ & & & & & & & & \end{array}$

\section{Clinical}

Presentation

$\begin{array}{llllllllll}\begin{array}{l}\text { Met MSIS } \\ \text { criteria }\end{array} & \begin{array}{l}50 \\ (88)\end{array} & \begin{array}{l}26 \\ (84)\end{array} & 0.747 & 38(95) & 12(71) & 0.747 & \begin{array}{l}22 \\ (96)\end{array} & 4(50) & 0.009 \\ \begin{array}{l}\text { Sinus Tract } \\ \text { present }\end{array} & \begin{array}{l}7 \\ (12)\end{array} & \begin{array}{l}7 \\ (23)\end{array} & 0.233 & 6(15) & 1(6) & 0.233 & 5(22) & 2(25) & 0.999 \\ \begin{array}{l}\text { Elevated ESR } \\ \text { or CRP }\end{array} & \begin{array}{l}41 \\ (72)\end{array} & \begin{array}{l}24 \\ (77)\end{array} & 0.622 & 27(68) & 14(82) & 0.576 & \begin{array}{l}17 \\ (74)\end{array} & 7(88) & 0.999 \\ \begin{array}{l}\text { Elevated } \\ \text { Synovial WBC }\end{array} & 33 & \begin{array}{l}19 \\ (58)\end{array} & 0.823 & 21(53) & 12(71) & 0.757 & \begin{array}{l}13 \\ (57)\end{array} & 6(75) & 0.999 \\ \begin{array}{l}\text { Elevated } \\ \begin{array}{l}\text { Synovial } \\ \% \text { PMN }\end{array}\end{array} & \begin{array}{l}31 \\ (54)\end{array} & \begin{array}{l}20 \\ (65)\end{array} & 0.377 & 20(50) & 11(65) & 0.358 & \begin{array}{l}14 \\ (61)\end{array} & 6(75) & 0.333 \\ \end{array}$

OA - osteoarthritis; IA - inflammatory arthritis; CP - culture positive; CN - culture negative; MSIS meets Musculoskeletal Infection Society diagnostic criteria

\section{Culture Negative PJI in Inflammatory Arthritis patients vs OA patients}

In a sub-analysis of CN-IA patients to CN-OA patients, fewer CN-IA scored positive for PJI based on PMN count, compared to $\mathrm{CN}-\mathrm{OA}$, but the difference was not significant $(p=0.08)$. IA-CN were younger than $O A$ $\mathrm{CN}$ (59 vs $69, p=0.01$ ). One-year survivorship free from infection of $\mathrm{CN}-\mathrm{OA}$ and $\mathrm{CN}-\mathrm{IA}$ was $87 \%$ and $66 \%$ respectively. CP-IA patients had the lowest one-year survivorship at $47 \%$. 


\section{Culture Positive PJI in Inflammatory Arthritis patients vs OA patients}

In a sub-analysis of CP-IA and CP-OA patients, there was no difference in diabetes, comorbidities, smoking, or history of PJI, but more CP-IA were female $(p<0.001)$, younger $(p<0.001)$ and used glucocorticoids ( $p<0.001$ ), as is typical for RA and SLE. Across all CP cases, $57 \%$ were staphylococcal, with no differences between groups. Overall surgical treatment included 2-stage exchange (55\%), DAIR $(39 \%)$ and 1-stage exchange (4.5\%), with no difference between groups $(p=0.8)$. There was a trend towards increased treatment failure for CP-IA, but the difference was not significant $(p=0.2)$.

\section{Culture Negative PJI vs Culture Positive PJl among Inflammatory arthritis patients}

Comparing CN-IA vs. CP-IA, no difference was observed in age, smoking, diabetes, or Charlson comorbidities, but there was a trend towards higher prevalence of prior PJI in the CN-IA group (Table 2). No differences were found in surgical treatment or use of biologics and DMARDs between CN and CP IA patients. A lower proportion of CN-IA had positive histology for acute infection, i.e. $\geq 10 \mathrm{PMN}$ per $5 \mathrm{HPFs}$ (0.003) and met MSIS criteria (0.009) than in CP-IA (Table 3). The majority of the IA-CN group (63\%) had signs of high chronic inflammation (scores 2 or 3 ) and only $25 \%$ had histological signs of acute infection compared to $87 \%$ of the IA-CP group $(p=0.003)$. The cases of IA with only chronic inflammation had typical perivascular and or interstitial chronic lymphocytic infiltrate, with differing amounts of plasma cells. They showed a minor component of PMN cells, although not reaching the cutoff for acute infection (Fig. 2).

\section{Kaplan-Meier Survivorship}

Overall IA survivorship free from infection reoperation was $52 \%$ at 1 year and $48 \%$ at 2 years. One-year survivorship of $\mathrm{CN}-\mathrm{IA}$, and $\mathrm{CP}-\mathrm{IA}$ were $66 \%$ and $47 \%$, respectively with no difference between survival curves, $p=0.163$ (Fig. 3). Overall culture negative survivorship free from infection reoperation was $85 \%$ at 1 year and $76 \%$ at 2 years. One-year survivorship of CN-OA was $87 \%$ with no difference in one-year survivorship when compared to CN-IA $(66 \%, p=0.36)$.

\section{Discussion And Conclusions}

In this retrospective pilot study, inflammatory arthritis patients with RA or SLE had a significantly higher rate of culture negative cases than OA patients. Furthermore, histopathology of surgical specimens revealed that a lower proportion of IA-CN patients had signs of acute infection, compared to the OA-CN cohort, and fewer IA-CN met MSIS criteria for PJI compared to OA-CN. In our cases, $75 \%$ of the IA-CN group did not have features of acute infection on histopathology and $50 \%$ did not meet MSIS criteria for infection, although all were treated as a PJI. Our findings support our hypothesis that some IA-CN PJI may have alternate non-infectious diagnoses, including flares of underlying inflammatory arthritis. 
To our knowledge, this is the first study comparing $\mathrm{CP}$ to $\mathrm{CN}-\mathrm{IA}$ and -OA patients while reviewing histopathology, and we demonstrate a statistically significant higher rate of culture negative PJI in the IA group (27.8\%) compared to a matched OA group (14.1\%). There is limited literature regarding culture negative PJI in IA cohorts. In the retrospective study by Berbari et al, looking at a cohort of 200 RA patients with PJI, they found only $9 \%$ were culture negative and RA was not an independent risk factor for culture negative PJI. ${ }^{14}$ In another retrospective analysis by Hseih et al, of 46 RA patients with PJI, none were culture negative. ${ }^{15}$ Finally, the paper by Schrama et al reports a $37 \%$ culture negative rate in the RA cohort of 49 patients, though they attribute this high percentage to possible prior courses of antimicrobial therapy. ${ }^{4}$ These studies, however, lack the histopathological analysis our study provides which supports our hypothesis as many IA-CN patients lack classic histological findings of bacterial PJI.

The incidence of $\mathrm{PJI}$ among patients with RA, and other forms of IA, is $50-80 \%$ greater than in OA patients. ${ }^{16}$ This higher risk may be attributed to immunosuppressive agents for RA patients such as glucocorticoids and disease modifying antirheumatic drugs (DMARDs). In this retrospective review, 75\% of the IA-CN group did not have features of acute infection on histopathology and $50 \%$ did not meet MSIS criteria for infection, although all were treated as a PJI by infectious disease and orthopedic specialists. A histological diagnosis is very important in the assessment of possible PJI, and forms part of the minor MSIS diagnostic criteria at a threshold of 5 PMNs /HPF. ${ }^{2}$ The AAOS recognizes that $5 \mathrm{PMN} / \mathrm{HPF}$ confers a likelihood ratio of $13.82(7.29,26.19)$; however, 10 PMN/HPF in 5 fields confers a likelihood ratio of $56.5(20.3,157.2) .{ }^{17}$ Given the high sensitivity and specificity of histopathology for PJI, it is important to note that $75 \%$ of IA-CN patients did not have positive histology and $63 \%$ had signs of lymphoplasmacytic chronic inflammation, a pattern which is classically seen in established rheumatoid arthritis, rather than in an infective process. ${ }^{18}$

As previously mentioned, a major limitation of this study is that half of our IA-CN cohort did not meet MSIS criteria for PJI but were included in the database because they were treated for PJI by the attending orthopedist and ID specialist. This supports our hypothesis that differentiating between a septic joint and an IA flare is challenging. Standard diagnostic tests, such as levels of serum and synovial white blood cell (WBC) counts and biomarkers such as erythrocyte sedimentation rate (ESR) and C-reactive protein (CRP), leukocyte esterase or alpha-defensin, may be similar in both and therefore may be misleading in the diagnostic process. A systematic literature review performed by Mirza et al to evaluate the efficacy of synovial biomarkers for diagnosing PJI in IA patients, showed that whilst the sensitivities are high for many serum and synovial tests, specificities in this patient population are low. ${ }^{11}$ They showed that although levels of synovial WBCs, IL-6, IL-8 and serum CRP appear higher in infected patients with IA, there is overlap with those who are not infected. The poor specificity of standard diagnostic tests contributed to the development of our hypothesis that a proportion of IA patients are not infected but are flaring.

Importantly, cases are included in the database through diagnosis and treatment by an attending orthopedist and ID specialist with expertise in the diagnosis and therapy of PJI, and not all cases meet 
MSIS criteria for PJI. However, we were intrigued by the high proportion of culture negative inflammatory arthritis patients in this small retrospective cohort, and given the overlap in available diagnostic tests found support for our hypothesis that some IA cases treated as PJI may in fact represent flares of IA. A more detailed prospective study with larger numbers is warranted to further study IA-CN patients who receive a presumptive diagnosis of PJI. New technologies, such as next generation sequencing (NGS), hold promise as a useful adjunct in the identification of an organism in culture-negative PJI, and may provide increased sensitivity in isolating organisms that cannot be identified using conventional culture. $^{19}$

Our study highlights a clinical issue that is pertinent to patients with IA who present with a single inflamed joint post arthroplasty. Little data are available to guide clinicians, and it remains challenging to manage these patients, particularly those who are culture negative. This preliminary work supports the need for further studies, not only looking at differences in clinical features between CN-IA and CP-IA PJI but also in assessing the discriminatory ability of biological diagnostic markers, are needed. Since rapid and accurate diagnosis of PJI is critical to ensure optimal outcomes a more robust PJI diagnostic algorithm aimed specifically at IA patients is needed.

\section{Declarations}

Ethics approval and consent to participate: all methods in this retrospective study were performed in accordance with the relevant guidelines. Hospital for Special Surgery's Institutional Review Board (ethics committee) approval was waived for this retrospective study prior to study initiation (study: \# 2016- 549). Informed consent was obtained from all subjects (all patients in this study are over 18 years of age).

Consent for publication: not applicable

Availability of data and materials: The datasets used and/or analysed during the current study are available from the corresponding author on reasonable request.

\section{Competing interests}

PS reports the following disclosures: EOS Imaging: Paid consultant; Intellijoint Surgical: Paid consultant; Lima Corporate: Paid consultant.

LD reports the following disclosures: Karius, Inc.: Research support; Stryker: Paid consultant. LR reports the following disclosure: Arthritis Foundation: Board or committee member.

MF reports the following disclosures: HS2: Stock or stock options; Insight: Stock or stock options; Lima: IP royalties, paid consultant; mekanika: Stock or stock options; Wishbone: IP royalties, paid consultant, stock or stock options.

SMG reports the following disclosures: Novartis: Paid consultant, Research support. 
CJM, MK, AOM, MH, AN, and TP have nothing to disclose.

Funding: not applicable

\section{Authors' contributions:}

PS: conceptualization, methodology, data collection, writing

MK: data curation, writing

CJM: project administration, formal analysis, writing, reviewing and editing

AOM: conceptualization, methodology, writing and editing

LD: methodology, original draft preparation

MH: conceptualization, methodology, reviewing and editing

LR: supervision, reviewing and editing

MF: conceptualization, data collection

AN: data analysis

TP: histological analysis and data analysis

SMG: supervision, writing original draft

Acknowledgements: not applicable

\section{References}

1. Fillingham YA, Della Valle CJ, Suleiman LI, et al. Definition of Successful Infection Management and Guidelines for Reporting of Outcomes after Surgical Treatment of Periprosthetic Joint Infection: From the Workgroup of the Musculoskeletal Infection Society (MSIS). J Bone Jt Surg - Am Vol. 2019. doi:10.2106/JBJS.19.00062

2. Parvizi J, Tan TL, Goswami K, et al. The 2018 Definition of Periprosthetic Hip and Knee Infection: An Evidence-Based and Validated Criteria. J Arthroplasty. 2018. doi:10.1016/j.arth.2018.02.078

3. George DA, Konan S, Haddad FS. Single-Stage Hip and Knee Exchange for Periprosthetic Joint Infection. J Arthroplasty. 2015. doi:10.1016/j.arth.2015.05.047

4. Schrama JC, Espehaug B, Hallan G, et al. Risk of revision for infection in primary total hip and knee arthroplasty in patients with rheumatoid arthritis compared with osteoarthritis: A prospective, population-based study on 108,786 hip and knee joint arthroplasties from the Norwegian arthroplast. Arthritis Care Res. 2010. doi:10.1002/acr.20036 
5. Ravi B, Escott B, Shah PS, et al. A systematic review and meta-analysis comparing complications following total joint arthroplasty for rheumatoid arthritis versus for osteoarthritis. Arthritis Rheum. 2012. doi:10.1002/art.37690

6. Chen $\mathrm{CH}$, Chen TH, Lin YS, et al. The impact of systemic lupus erythematosus on the risk of infection after total hip arthroplasty: A nationwide population-based matched cohort study. Arthritis Res Ther. 2020. doi:10.1186/s13075-020-02300-1

7. Burn E, Edwards CJ, Murray DW, et al. The impact of rheumatoid arthritis on the risk of adverse events following joint replacement: A real-world cohort study. Clin Epidemiol. 2018. doi:10.2147/CLEP.S160347

8. Premkumar A, Morse K, Levack AE, Bostrom MP, Carli A V. Periprosthetic Joint Infection in Patients with Inflammatory Joint Disease: Prevention and Diagnosis. Curr Rheumatol Rep. 2018. doi:10.1007/s11926-018-0777-6

9. Reisener M, Perka C. Do Culture-Negative Periprosthetic Joint Infections Have a Worse Outcome Than Culture-Positive Periprosthetic Joint Infections? A Systematic Review and Meta-Analysis. Biomed Res Int. 2018. doi:10.1155/2018/6278012

10. Tan TL, Kheir MM, Shohat N, et al. Culture-Negative Periprosthetic Joint Infection: An Update on What to Expect. JB JS open access. 2018. doi:10.2106/JBJS.0A.17.00060

11. Mirza SZ, Richardson SS, Kahlenberg CA, et al. Diagnosing Prosthetic Joint Infections in Patients With Inflammatory Arthritis: A Systematic Literature Review. J Arthroplasty. 2019. doi:10.1016/j.arth.2019.01.051

12. Quan H, Sundararajan V, Halfon P, Fong A. Coding algorithms for defining comorbidities in. Med Care. 2005.

13. Martin Bland J, Altman DG. Survival probabilities (the Kaplan-Meier method). Br Med J. 1998.

14. Berbari EF, Osmon DR, Duffy MCT, et al. Outcome of Prosthetic Joint Infection in Patients with Rheumatoid Arthritis: The Impact of Medical and Surgical Therapy in 200 Episodes. Clin Infect Dis. 2006. doi: $10.1086 / 498507$

15. Hsieh PH, Huang KC, Shih HN. Prosthetic Joint Infection in Patients with Rheumatoid Arthritis: An Outcome Analysis Compared with Controls. PLoS One. 2013. doi:10.1371/journal.pone.0071666

16. Yeganeh MH, Kheir MM, Shahi A, Parvizi J. Rheumatoid Arthritis, Disease Modifying Agents, and Periprosthetic Joint Infection: What Does a Joint Surgeon Need to Know? J Arthroplasty. 2018. doi:10.1016/j.arth.2017.11.031

17. Parvizi J, Delia Valle CJ. AAOS clinical practice guideline: Diagnosis and treatment of periprosthetic joint infections of the hip and knee. J Am Acad Orthop Surg. 2010. doi:10.5435/00124635201012000-00007

18. Hanaoka BY, Ithurburn MP, Rigsbee CA, et al. Chronic Inflammation in Rheumatoid Arthritis and Mediators of Skeletal Muscle Pathology and Physical Impairment: A Review. Arthritis Care Res. 2019. doi:10.1002/acr.23775 
19. Tarabichi M, Shohat N, Goswami K, et al. Diagnosis of periprosthetic joint infection: The potential of next-generation sequencing. J Bone Jt Surg - Am Vol. 2018. doi:10.2106/JBJS.17.00434

\section{Figures}
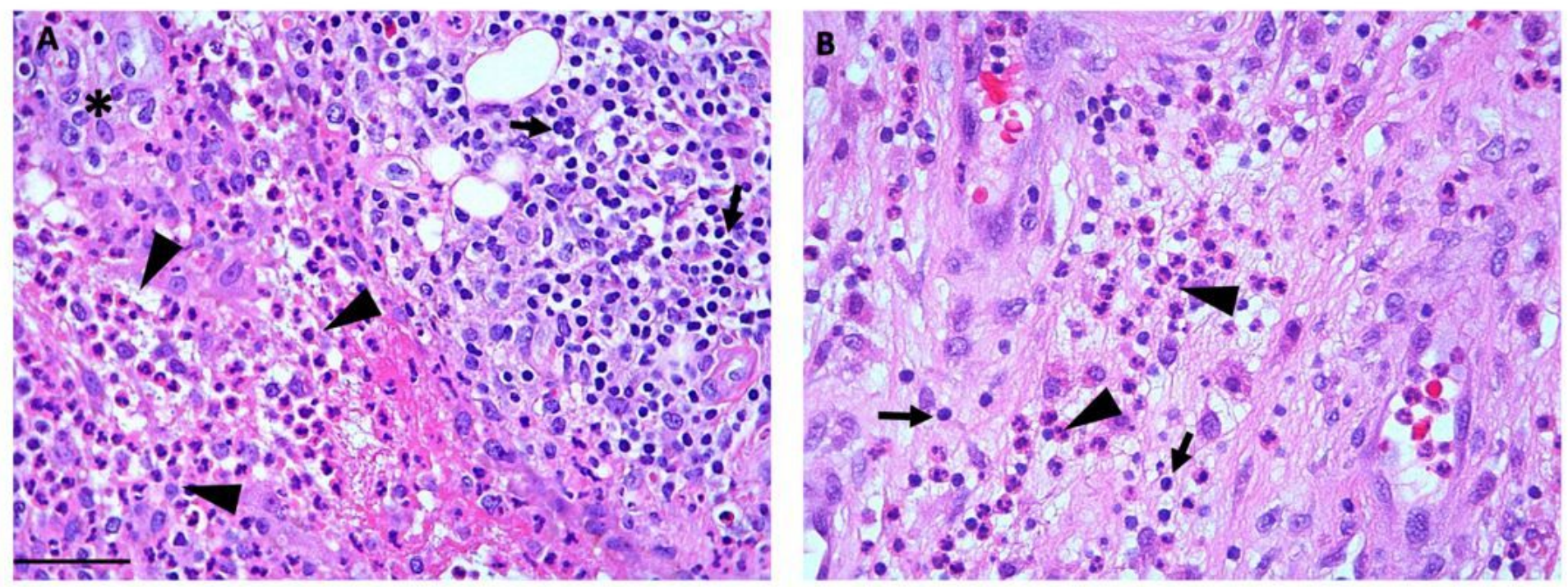

Figure 1

H\&E showing high field image with more than 10 PMNs (arrowheads). A) Shows a dense infiltrate of lymphocytes(arrows) and monocytes (*). B) Shows the majority of inflammatory cells are granulocytes, and there is a minor component of sparse lymphocytes (arrows). (400x magnification. Bar $=50 \mu$ )
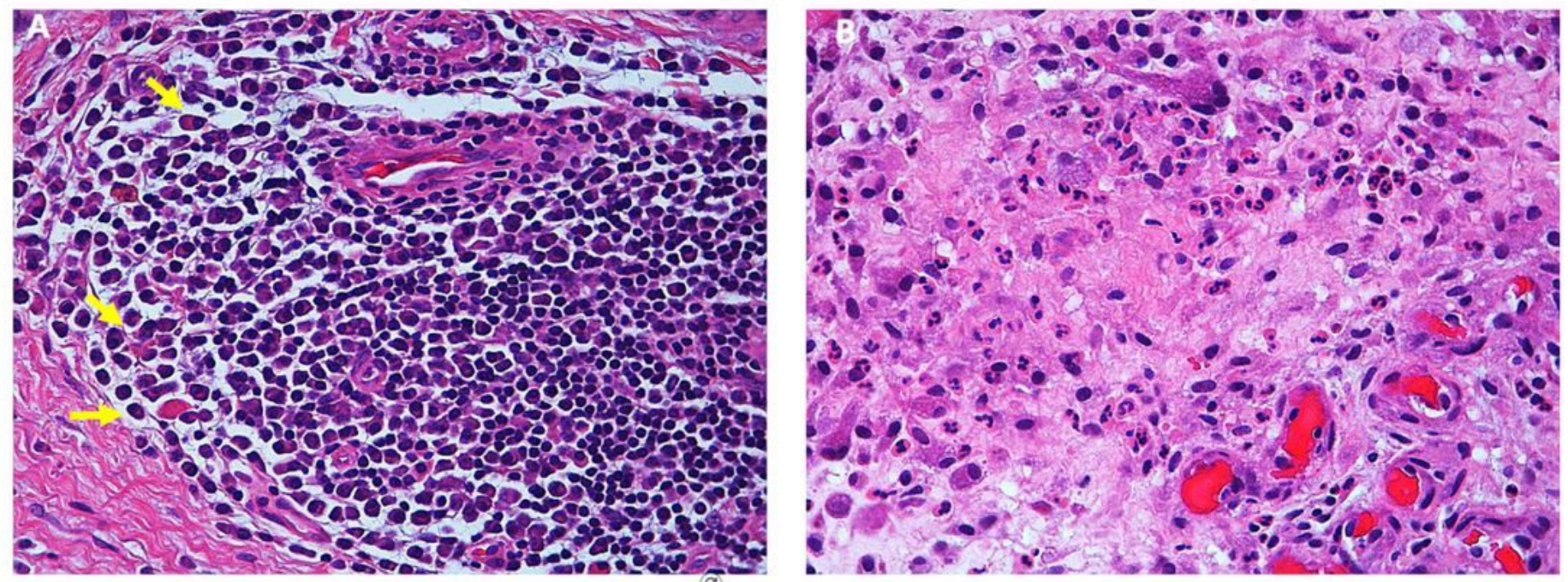

Figure 2

An Example of chronic inflammation in IA-CN case, demonstrating heavy perivascular chronic inflammation, including numerous plasma cells (arrows). PMNs are above the threshold in a few fields (B), but do not meet the 5 fields cutoff. (200x magnification. Bar $=50 \mu$ ) 


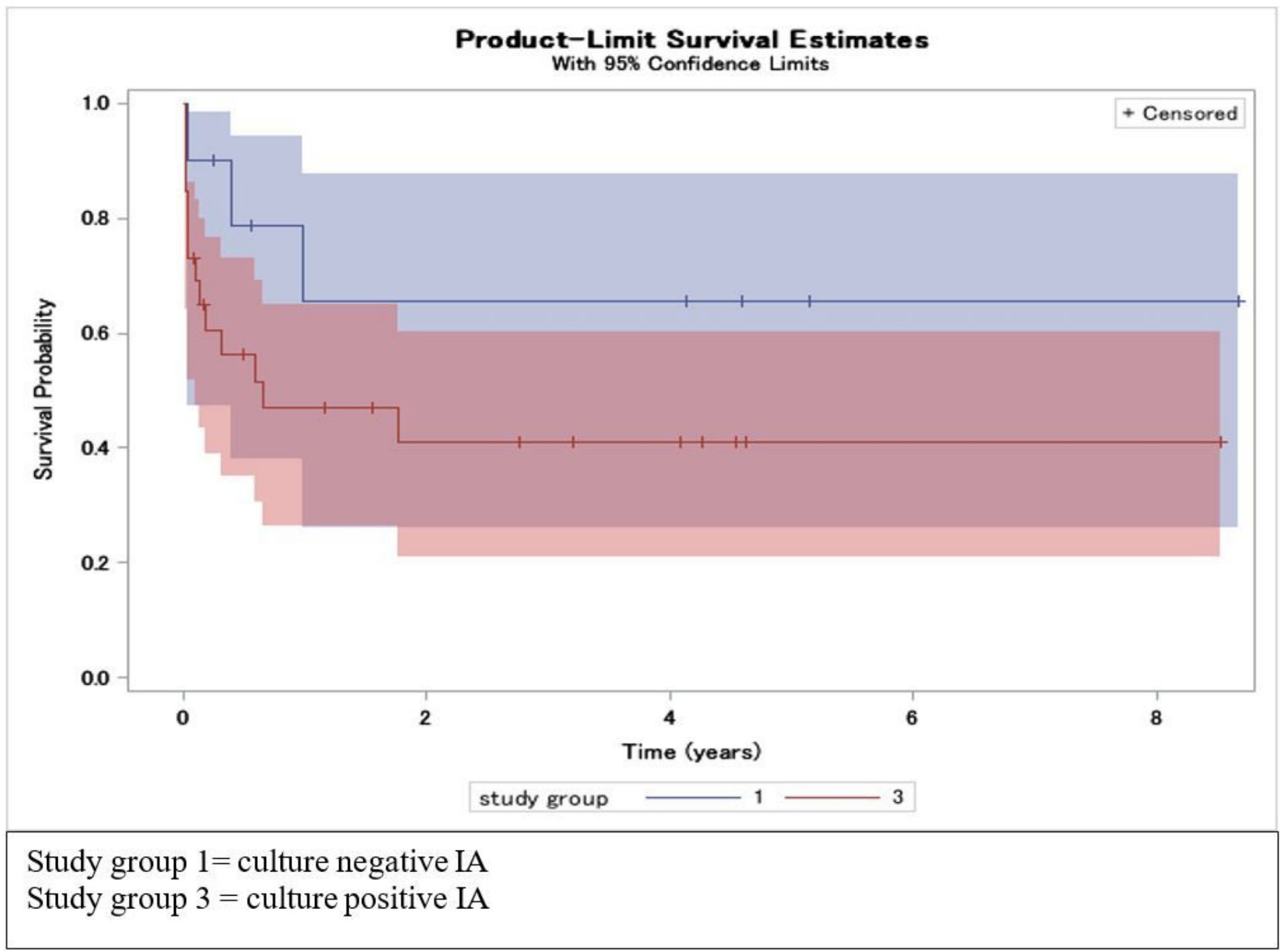

\section{Figure 3}

Kaplan-Meier survivorship curves (with 95\% Cls) with survival defined as treatment success free from infection reoperation at 2 years. 